\title{
Le musée de Minéralogie - MINES ParisTech
}

\section{Didier Nectoux}

\section{(2) OpenEdition \\ 1 Journals}

Édition électronique

URL : http://journals.openedition.org/artefact/1605

DOI : 10.4000/artefact.1605

ISSN : 2606-9245

Éditeur :

Association Artefact. Techniques histoire et sciences humaines, Presses universitaires du Midi

Édition imprimée

Date de publication : 30 mai 2018

Pagination : 247-252

ISBN : 978-2-7535-7494-6

ISSN : 2273-0753

Référence électronique

Didier Nectoux, «Le musée de Minéralogie - MINES ParisTech », Artefact [En ligne], 7 | 2017, mis en ligne le 14 février 2019, consulté le 14 septembre 2020. URL : http://journals.openedition.org/artefact/ 1605

\section{(c) (i) $\odot$}

Artefact, Techniques, histoire et sciences humaines est mise à disposition selon les termes de la Licence Creative Commons Attribution - Pas d'Utilisation Commerciale - Pas de Modification 4.0 International. 


\section{Le musée de Minéralogie - MINES \\ ParisTech}

Didier NECTOUX*

Le musée de Minéralogie de l'École des mines ParisTech est situé au pied de la montagne Sainte-Geneviève, en plein cœur du quartier latin. L'École et le cabinet des mines se sont installés dans l'hôtel de Vendôme en août 1815. Il fallut attendre le milieu du xIx ${ }^{\mathrm{e}}$ siècle, après que l'État eut acquis en 1838 les bâtiments, pour que les collections soient rangées et présentées dans des meubles et vitrines en chêne de Hongrie. Cet ensemble de pièces en enfilade et son mobilier ont été conservés dans la configuration de l'époque (1850 à 1856) (Fig. XXX, cahier couleur). Ce lieu, épargné par le temps, est un véritable écrin des merveilles minérales. Plus de 4000 échantillons sont exposés dans des vitrines verticales et horizontales réparties dans les différents salons : la salle d'entrée, le salon Haüy et les différentes salles de la galerie de 80 mètres de long dont les baies s'ouvrent sur le jardin du Luxembourg.

\section{Des collections pour quoi faire?}

L'histoire du musée de Minéralogie est intimement liée à celle de l'École des mines créée le 19 mars 1783 par un arrêté de création du Conseil d'État du roi Louis XVI. Cependant les minéraux et échantillons nécessaires pour les enseignements de docimasie et de Minéralogie sont la propriété de Balthazar George Sage (1740-1824), premier directeur de l'école. Ils resterontà l'hôtel dela Monnaie ou l'école débuta, jusqu'à la mort de ce dernier. Le cabinet de Minéralogie est officiellement institué lors de la nomi- nation de René Just Haüy (1743-1822), premier conservateur, en octobre 1794. Les échantillons de minéraux, de roches, de minerais vont alors s'accumuler au fil des années par dizaines de milliers. Ils seront analysés puis classés par gisements, départements, pays. Les collections s'enrichiront par des achats de collections privées (de Drée, de Dietrich, Sage) mais aussi des dons et des collectes de géologues célèbres comme Werner, Brongniart, Berthier, Dufrenoy, Daubrée, Mallard, Termier et tant d'autres grands

\footnotetext{
*. Didier Nectoux est Conservateur du musée de Minéralogie MINES Paris Tech.
} 
scientifiques ou modestes amateurs collectionneurs.

Cet ensemble se compose à ce jour de près de 100000 objets et échantillons et forme une unité qui se place au quatrième rang mondial. Le musée de Minéralogie offre au regard du visiteur, sur près de $1000 \mathrm{~m}^{2}$, les plus belles pièces des collections de minéraux, de roches, de météorites, de pierres précieuses brutes et taillées (dont certaines issues des joyaux de la Couronne de France).

On devine aisément que le rôle de ces collections était pédagogique, en vue de former des ingénieurs des mines. Pourtant dès leur création, l'État français (décret du Comité de Salut Public du 12 juillet 1794) leur a attribué un intérêt stratégique. Elles devaient constituer un inventaire des richesses minéralogiques de la France et de ses possessions en vue de leur exploitation pour les mettre au service de l'industrie naissante (Fig. XXXII, cahier couleur). Ce rôle a perduré pendant le $\mathrm{XIX}^{\mathrm{e}}$ siècle puis s'est progressivement estompé pendant le $\mathrm{xx}^{\mathrm{e}}$ siècle. Aujourd'hui, le développement des nouvelles technologies et leurs besoins en terres et éléments rares a réactivé cette mission.

Le musée de Minéralogie n'est plus seulement un conservatoire des espèces et une vitrine de la richesse et la beauté du monde minéral. Il est aussi un lieu de recherche. En effet, depuis ces dernières années, on découvre de plus en plus d'utilisations industrielles aux éléments contenus dans les minéraux. Les nouvelles technologies font appel aux terres rares et à des éléments, par exemple le Tantale, le Niobium ou le Germanium, pour fabriquer les téléphones portables, les panneaux photovoltaïques, les aciers spéciaux pour l'aéronautique ou l'aérospatiale. Il est donc nécessaire de revisiter l'ensemble des gisements qui sont, ou ont été, exploités pour connaître leurs potentialités et leurs richesses au regard de ces nouveaux usages. Les collections de minéraux deviennent ainsi de véritables banques de données que les chercheurs analysent afin de définir des stratégies en matière d'acquisition et d'exploitation des ressources minérales. Aux siècles derniers les gisements semblaient infinis et inépuisables. Aujourd'hui on sait que ce n'est pas le cas et que les grands enjeux de notre civilisation portent sur l'exploitation raisonnée et la bonne gestion des ressources naturelles pour assurer le développement des sociétés, tout en préservant le mieux possible l'environnement. Le musée de Minéralogie contribue à sa manière à cette démarche et, fabuleux trésor des siècles passés, s'inscrit résolument dans les débats qui animent la société contemporaine.

\section{De nouveaux objectifs pour le musée}

La mondialisation, la croissance de la population mondiale, l'emballement de la consommation et leurs impacts sur l'environnement font actuellement l'objet de débats et affrontements planétaires. Les politiques et le grand public sont aujourd'hui avides d'informations sur les matières premières minérales, leur 
exploitation et leur transformation pour alimenter la réflexion. Les Écoles des mines et leurs musées sont sans doute les mieux placés pour apporter une connaissance technique et scientifique sur le sujet. La prise de conscience par nos dirigeants, bien que tardive car initiée ces dernières années, se traduit par une vraie volonté de s'ouvrir au plus grand nombre et le désir de rendre attractif ce lieu préservé du temps qu'est le musée de Minéralogie mines ParisTech. Les paragraphes qui vont suivre ont pour but de présenter et développer les objectifs et premières réalisations concrètes du musée dans ce contexte qui constitue une véritable opportunité de développement jamais rencontrée jusqu'alors.

\section{Jouer un rôle sociétal}

Les enjeux environnementaux, stratégiques, économiques et politiques liés à l'exploitation des matières premières minérales, hier importants, sont aujourd'hui devenus vitaux. Mais le citoyen manque d'informations scientifiques et techniques pour débattre le plus rationnellement possible des choix et compromis qui doivent être faits pour concilier développement économique et protection de l'environnement. Le musée de Minéralogie a pour objectif de devenir à la fois un lieu de découverte des processus industriels qui transforment les minéraux en objets manufacturés et un lieu de débats sur ce qui doit être fait en matière d'environnement pour un développement raisonné de nos sociétés.

\section{Être une vitrine de l'industrie et de l'artisanat}

Établir un lien entre les minerais, les minéraux et les produits manufacturés se concrétise à travers des expositions thématiques temporaires. Ces aspects sont systématiquement abordés dans les circuits de visites organisées. Des publications écrites (livre "Curiosités minérales ») et audiovisuelles (un MOOC - Massive Open Online Course "Roches et minéraux courants », de courtes vidéos « Histoires de cailloux » sur les réseaux sociaux) viennent appuyer le discours et toucher plus largement le grand public. Les partenariats entre une entreprise/une fédération pro- fessionnelle se déclinent en différentes actions : la mise en place d'expositions et publications associées, la restauration et l'entretien des locaux et mobiliers, ou l'acquisition de minéraux exceptionnels pour l'enrichissement de la collection, l'organisation de visites ou de réceptions, destinées aux salariés, clients ou partenaires de l'entreprise. Selon leur objet, ces coopérations se font dans le cadre de conventions de mécénat, qui rendent le donateur éligible à une réduction d'impôt de $60 \%$ du montant versé, et qui sont signées avec l'École mines ParisTech et la Fondation mines 
ParisTech, ou de conventions de gré à gré, signées avec l'École.

\section{Être une vitrine de l'École et un lieu d'échange culturel}

Au début $\mathrm{du} \mathrm{xxI}^{\mathrm{e}}$ siècle, à l'heure de la mondialisation, les grandes écoles d'ingénieurs françaises ont vu leurs positions changer au regard de l'émergence de grandes universités étrangères regroupant des dizaines de milliers d'étudiants. Aujourd'hui, les grandes écoles et les institutions se regroupent. L'École des mines est actuellement associée à l'Institut Mines-Télécom et s'est regroupée sous l'égide de l'association ParisTech et s'est intégrée à la COMUE des établissements d'enseignement des sciences et techniques mais aussi d'économie et d'arts et lettres. Le musée de Minéralogie a, dans ce contexte, un double rôle à jouer : celui stabilisateur, qui ancre dans l'histoire et le passé une institution, et participe à son identité et sa légitimité; celui d'agent de liaison en établissant des liens culturels avec le grand public et en suscitant des projets et actions transdisciplinaires (arts et sciences) entre les grandes institutions parisiennes.

\section{Communiquer sur les minéraux "Beaux et utiles»}

Le livre «Curiosités minérales », paru en décembre 2013, réédité en 2017, présente les collections à travers de somptueuses photographies et des anecdotes qui pour la plupart traitent des utilisations industrielles des minerais et des éléments qu'ils contiennent.

Mineralotech, la page Facebook et le compte Twitter du musée, reprennent ces anecdotes à raison de deux publications par semaine. La déclinaison du concept ne s'arrête pas là. Il existe aussi une exposition itinérante, "Cristaux » qui déjà été accrochée aux grilles de l'ESPCI et de la maison de la chimie et installée dans les écoles de Poissy. Durant l'année 2014, une dizaine de conférences, " les minéraux sont beaux et utiles ", a été donnée à travers la France.

Les visites du musée de Minéralogie sont systématiquement orientées sur les gisements des minéraux, leur transformation et utilisations dans l'industrie. Ce parti pris en fait un véritable musée de l'industrie minérale tel qu'il était nommé au milieu du xIx ${ }^{\mathrm{e}}$ siècle. De la pharmacie à l'automobile ou l'aéronautique, les secteurs de l'industrie sont ainsi évoqués en présentant les minéraux classés par familles chimiques. Pour aider le visiteur individuel dans sa progression, dans le musée un certain nombre d'échantil- 
lons sont associés avec des QR Codes. À l'aide de son portable ou sa tablette, il peut accéder aux explications écrites et enregistrées liées aux minéraux. À court terme le visiteur se verra proposer des visites thématiques lui permettant d'aller d'un point à un autre en fonction de son choix. À titre d'exemples : les minéraux d'un pays, les minerais d'un métal, les minéraux présents dans un objet du quotidien, etc. Des vidéos illustrant les gisements ou les procédés industriels de fabrication viendront prochainement compléter les photographies, textes et enregistrements audio déjà existants.

\section{Du bâtiment au bijou de luxe}

Pour les minéraux utiles à la construction, une fiche thématique (cinq minéraux pour construire sa maison) est déjà proposée au visiteur afin d'identifier les liens entre minéral et produit manufacturé. Mais le principal vecteur de médiation est aujourd'hui un MOOC: « Roches et minéraux courants, Identification, propriétés et utilisations » dont la quatrième session a eu lieu en janvier 2017. Les sessions ont vu l'inscription de plus de 10000 internautes. Les vidéos tournées à cette occasion dans les carrières et sites industriels seront prochainement proposées au visiteur du musée sur les écrans disponibles mais aussi sur les applications portables et tablettes.

Avec sa collection de gemmes et pierres taillées, et la présentation de pièces issues des joyaux de la Couronne de France, le musée suscite l'intérêt des milieux de la bijouterie et de l'industrie du luxe. Les minéraux à l'état brut entrent en résonnance avec les objets précieux réalisés par les plus grands orfèvres et joaillers de la planète. De grandes maisons comme Jaquet Droz, Margiela ont sollicité le musée pour présenter leurs nouvelles collections. Deux expositions, à Dubaï et à Hong Kong ont été présentées en partenariat avec l'École des arts joailliers (Maison Van Cleef et Arpels) en 2017.

Développer cet axe de collaboration s'impose comme une évidence. La beauté du décor et des minéraux présentés (Fig. XXXI, cahier couleur) peuvent faire du musée de Minéralogie un lieu didactique, écrin des savoir-faire de la joaillerie.

\section{Conclusion}

Les points abordés dans cette rapide présentation illustrent la nouvelle dynamique qui anime le musée de Minéralogie mines ParisTech et son équipe. Cet effort doit cependant s'inscrire dans une démarche collective nationale et internationale pour qu'elle puisse être réellement audible et s'intégrer dans une démarche éducative et pédagogique. Il existe dans ce domaine des réseaux forts 
et structurés notamment dans les grands pays producteurs de métaux et de minéraux industriels comme le Canada ou les pays d'Amérique latine. Sur le continent européen, les initiatives existent déjà depuis de nombreuses années mais sont le fait de structures, musées ou associations isolées. Depuis juin 2016, Le réseau d'excellence, Rex « Mines et Société » qui fédère écoles (Mines Nancy, Mines Alès, ENSG, Mines Paris), industriels, fédérations professionnelles et associations, s'est attaché à développer des recherches, des formations et des opérations de communication qui augmentent l'activité et la visibilité du secteur des ressources minérales. Les productions du musée de Minéralogie ont été intégrées immédiatement dans cette démarche de vulgarisation des sciences de la terre et de valorisation des collections ${ }^{1}$.

\section{Notes}

1. Pour visiter les musées de Minéralogie : 60 boulevard Saint-Michel, 75006 Paris. Heures d'ouverture : du mardi au vendredi : 13h30-18h- ; samedi : 10h-12h30 et 14h17h. Tél. : (33 1) 40519139 et fax : (33 1) 40519175. Courriel : ] musee@mines-paristech.fr] ; site Internet : [http: / / www.musee.mines-paristech.fr/]. 